\title{
PROTOCOL FOR A MEDIATED LONG-TERM EXPERIMENT WITH A Social Robot
}

A PREPRINT

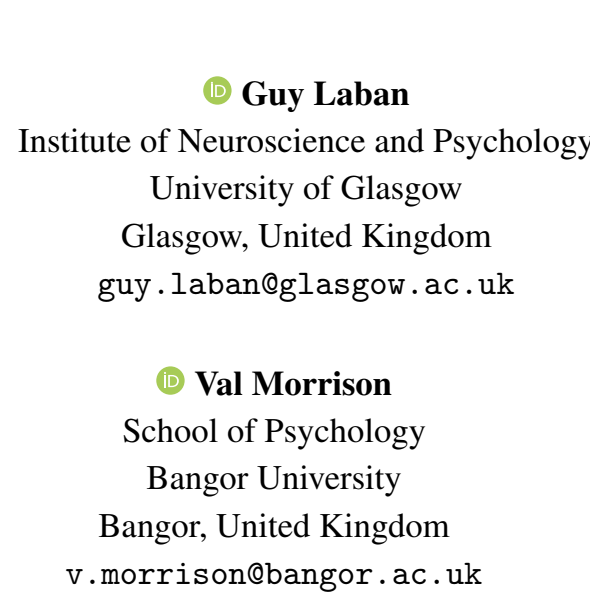

\author{
(1) Arvid Kappas \\ Department of Psychology and Methods \\ Jacobs University \\ Bremen, Germany \\ a.kappas@jacobs-university.de \\ Emily S. Cross* \\ Department of Cognitive Science \\ Macquarie University \\ Sydney, New South Wales, Australia \\ Institute of Neuroscience and Psychology \\ University of Glasgow \\ Glasgow, United Kingdom \\ emily.cross@mq.edu . au
}

\begin{abstract}
One major challenge faced by human-robot interaction (HRI) researchers is replicating and extending new findings, to better understand how short, constrained laboratory manipulations might translate to real-world scenarios. Since interactions with social robots are novel and exciting for many people, one particular concern is the extent to which people's behavioural and emotional engagement with robots might develop from initial interactions with a robot, when a robot's novelty is especially salient, and be sustained over time. The aim of this paper is to introduce a research protocol for a mediated long-term online experiment for testing the extent to which social robots' cognitive architectures can elicit emotionally rich disclosures and expressions from people to identify their needs and emotional states over time. Moreover, this protocol introduces experimental methods for investigating people's perceptions of a social robot in their natural settings during prolonged and regular interactions, and evaluating how novelty effects on human-robot interactions change over time.
\end{abstract}

Keywords Social Robots · Human Robot Interaction · Experimental Methodology and design · Longitudinal · Research protocol $\cdot$ Affective science $\cdot$ Self-disclosure $\cdot$ Communication

\section{Introduction}

Social robots can elicit socially meaningful behaviours and emotions from humans across a number of experimental and real-world contexts $(37,34,48)$. Nevertheless, one of the challenges to human-robot interaction (HRI) research is replicating and extending lab-based findings to better understand how short, constrained laboratory manipulations might translate to real-world scenarios. Since interactions with social robots are novel and exciting for many people, one particular concern in this specific area of HRI is the extent to which behavioural and emotional expressions might 
develop from initial interactions with a robot, when its novelty is particularly salient, to responses and behaviours that are sustained over time (70).

One of the challenges of studying novelty effects in HRI is that empirical studies in this area are often limited to controlled laboratory settings due to various logistical (e.g., limited number of robots per lab and robots' high cost) and technical factors (e.g., multiple computers or other controlling devices required to coordinate a robot's behaviours and/or requirements for skilled Wizard-of-Oz operation). These challenges can make it difficult for HRI researchers to gain insights into factors that shape people's long-term interactions with social robots in natural, real-world settings. This is especially noticeable with studies that are focused on evaluating the use and utility of social robots in social settings. Robots for these settings are often designed to interact and communicate with humans or other agents (such as pets or other robots) by following social scripts and rules relevant to their role and function within social settings (10, 34). Our understanding of social robots' potential scope and limitations will be substantially informed by devising experiments where people can interact with robots in more natural social settings, such as at home, one's workplace, or school.

In the current paper, we introduce a research protocol for a mediated long-term online experiment, aimed to test the extent to which social robots' cognitive architectures can elicit emotionally rich disclosures and expressions from people in their own home settings to identify their needs and emotional states over time. Moreover, the protocol aims to establish experimental methods for investigating people's perceptions of a social robot in natural everyday settings during prolonged and intense conversational interactions, and to measure participants' susceptibility to novelty effects.

\section{Methods}

Consistent with recent proposals (59, 69), we report how we determined our sample size, all data exclusions, all manipulations and all measures in the study. In addition, following open science initiatives (e.g., 57), the de-identified data sets, stimuli and analysis code associated with this study are going to be freely available online (https://osf.io/r5xga). By making the data available, we enable and encourage others to pursue tests of alternative hypotheses, as well as more exploratory analyses.

\subsection{Experimental Design}

A 2 (Discussion Theme: Covid-19 related or general) by 10 (chat sessions across time) between-groups repeated measures experimental design was followed. Participants were randomly assigned to one of the two discussion topic groups, according to which they conversed with the robot Pepper (SoftBank Robotics) via Zoom video chats about general everyday topics (e.g., social relationships, work-life balance, health and well-being; see Table 11). One group's conversation topics were framed within the context of the Covid-19 pandemic (e.g., social relationships during the pandemic, sustaining mental health during the pandemic, etc.), whereas the other group's conversation topics were similar, except that no explicit mention of the Covid-19 pandemic was ever made (see example at Section 2.4 .3 and table 27. Each interaction consisted of the robot asking the participant 3 questions (x3 repetitions). The topic of each interaction was assigned randomly before the experimental procedure started, as was the order of the questions. Participants were scheduled to interact with the robot twice a week during prearranged times, and each interaction with the robot lasted between 5 to 10 minutes, and another 10-20 minutes were taken up completing questionnaires. These interactions took place across five weeks between 25/02/21 and 02/04/21.

\subsection{Participants}

\subsubsection{Sample}

A priori power calculations using $G^{*}$ power software $(27,28)$ suggest that for reasonable power $(0.83)$ to detect small to medium effect sizes, a sample size of 22 participants would be required. Due to the relatively complex data collection 
procedure and the potential for a high dropout rate, we recruited 40 participants via Prolific website. One participant dropped out, resulting in a final sample size of 39 participants.

\subsubsection{Target Population}

The target population for this study is exclusively adults from the general population aged 18 or over with normal to corrected to normal vision, no known mental disability, hearing loss or difficulties, or physical handicap, are native English speakers, and currently reside in Great Britain. Due to the technical requirements of the mediated experimental design, the target population of this study consist of individuals with access to a personal computer with Zoom installed, a functioning web camera, stable internet connection, microphone, and speakers/headphones.

\subsubsection{Recruitment}

Participants were recruited via Prolific and were allowed to participate only after confirming that they were older then 18 years, are native English speakers, and have access to a computer with Zoom installed as well as a decent web camera, stable internet connection, microphone, and speakers/headphones. Also, Prolific users were asked to commit to attending 2 sessions a week across a 5 week period. Specific Prolific filters used for participant recruitment were as follows:

- Native language (being English).

- Age (18+).

- Current country of residence (being the United Kingdom).

- Place of most time spent before turning 18 (being either "In England" or "Elsewhere in the UK").

- Vision (reporting to have normal to corrected to normal vision).

- Hearing difficulties (reporting to not suffer from hearing loss or difficulties).

- Mild cognitive impairment/Dementia (reporting to never being diagnosed with mild cognitive impairment or dementia).

- Mental health/illness/condition - ongoing (reporting to not having or have had in the past a diagnosed, on-going mental health/illness/condition).

- Webcam (reporting to have a webcam or built-in camera and willing to use it as part of the study).

- Video call interview (reporting to be willing to participate in a face to face video call interview).

- Record video (reporting to be willing to be recorded via a webcam as part of a study).

- Participants were limited to complete the study on their desktop computer and could not participate in the study using mobile or tablet devices.

Since a longitudinal study is depended on participants' cooperation, the following filters were also applied in Prolific:

- Prolific tasks' approval rate (between $80 \%$ to $100 \%$ ).

- Number of previous submissions on Prolific (at least 15 completed submissions on Prolific).

After applying these filters, 4424 matching eligible Prolific users had been active in the past 90 days before starting the recruitment procedure, on Friday, 19/02/2021. Eligible Prolific users could access the Prolific page of the study to receive further information, consider their participation, and complete the induction questionnaire if interested. On the Prolific page of the study (of the induction questionnaire - Session 0) and in the induction questionnaire Qualtrics form, Prolific users were introduced to the study, the task, and the available time slots as part of the longitudinal experiment schedule. After receiving this information about the study's requirements, Prolific users were then asked if they would 
like to continue in the study by declaring that they can commit to the study's requirements. Following this, Prolific users were asked to sign a participant consent form. Prolific user then were asked to provide, if they wished, optional consent to allow the research team to use their video and audio footage (including videos, audios, and photos made from video material) as materials for research publications, conference presentations, and other multimedia outputs that can and might be disseminated and distributed online, in the media and for public presentations. Finally, Prolific users were then asked to choose their participation time slots, after which they received a participant number to start their participation. They were then immediate able to begin answering the induction questionnaire (Session 0; see more information about the procedure at section 2.5.3.

Participants were paid a total of $£ 3$ for every 30 minutes of participation or participation session if it lasted less than 30 minutes. Participants who completed all 10 sessions were paid an extra $£ 20$ after their final interaction. From the 4424 matching eligible Prolific users on Prolific, 40 participants were recruited on Friday, 19/02/2021, to start their participation by completing an induction questionnaire (Session 0) using a Qualtrics form. After completing the induction questionnaire, the last page of the form reminded participants of their participation time slot and day, and the date for their first session in the experimental procedure (Session 1) after the induction session (Session 0). The first interaction with the social robot as part of the experiment (Session 1) took place on Thursday, 25/02/21, for those who scheduled their sessions on Mondays and Thursdays, and on Friday, 26/02/21, for those who scheduled their sessions on Tuesdays and Fridays.

\subsubsection{Ethics and Communication}

All study procedures were approved by the research ethics committee of the University of Glasgow (ethics approval number: 300200132). As described in the previous section, all participants provided written informed consent before participating in the study. All Prolific users interested in participating in the study were introduced to the study, the requirements of the study, and the task, but were not informed about the functionalities of the robot Pepper, in order to ensure all robot knowledge or priming was minimised. During each session (including Session 0), participants were re-introduced to the study, the study's schedule (in relation to their chosen day of participation), and received reminders and information about what the study involves. Furthermore, they were reminded about the benefits and risks of their participation (i.e., ensuring that they would receive their payment, no risks were anticipated as a result of study involvement, and their right to withdraw their participation at any time with no penalty or punishment). Participants were further informed how their data (i.e., behavioural and self-reported data collected in the study) would be used and again reminded of their right to withdraw their data and/or ask that it not be used at any time during or after their participation. Participants were guaranteed that their right to privacy and anonymity would be respected and that no identifiable data would be shared with anyone beyond the research team. Participants were reminded that their participation was voluntary and they were given the contact information of the main researcher and experimenter (GL) should they wish to follow up with any further questions. After completing the study, participants received a comprehensive debriefing message in Prolific (forwarded by Prolific to their associated email address), providing further information about the study, the deception that was used (i.e., the experimenter was using Wizard of Oz (WoZ) approach for communicating with participants in order to make it look like the robot was responding autonomously), and were again given the contact information of the main researcher and experimenter (GL) should they wish to follow up with any further questions or feedback.

When completing each session, participants were reminded in the Qualtrics form about date of their next session. Two days before each session, participants received an email via Prolific regarding the specifics of their next session. This message contained details about the session number, the time in which the Prolific page with the link for the session questionnaire form would be published, a reminder not to start the session before the allocated participation time slot, and to contact the experimenter (GL) if they are to be late, cannot remember their participation time-slot, or cannot make it. Finally, participants were thanked for their participation and cooperation in each of these messages and were reminded of their rights and the fact that they were welcome to contact the experimenter (GL) at any time using the 
Prolific messaging system, or by email. On the day of participation, participants received an automated message by Prolific at 08:00 AM, that the Prolific page of the session is available online. Later that day, 30 to 15 minutes before each participant's participation time slot, each participant received an individual message via Prolific from the experimenter (GL) about their upcoming session and where they could find the link to start the session. If and when participants were late to their participation (without providing earlier notice that this would be the case), the experimenter (GL) messaged the participant via Prolific to ensure attendance or rescheduling the session. When participants experienced any technical difficulties or needed to communicate with the experimenter, they were instructed to do this via Prolific or email, and not using the Zoom chat. This was to reduce any potential association between the session interactions with the robot and the experimenter. Accordingly, all communications between participants and the experimenter took place via the Prolific messaging centre, or emails on rare occasions (when initiated by a participant). The main researcher and experimenter (GL) signed his name on all communications with participants.

\subsection{Stimuli}

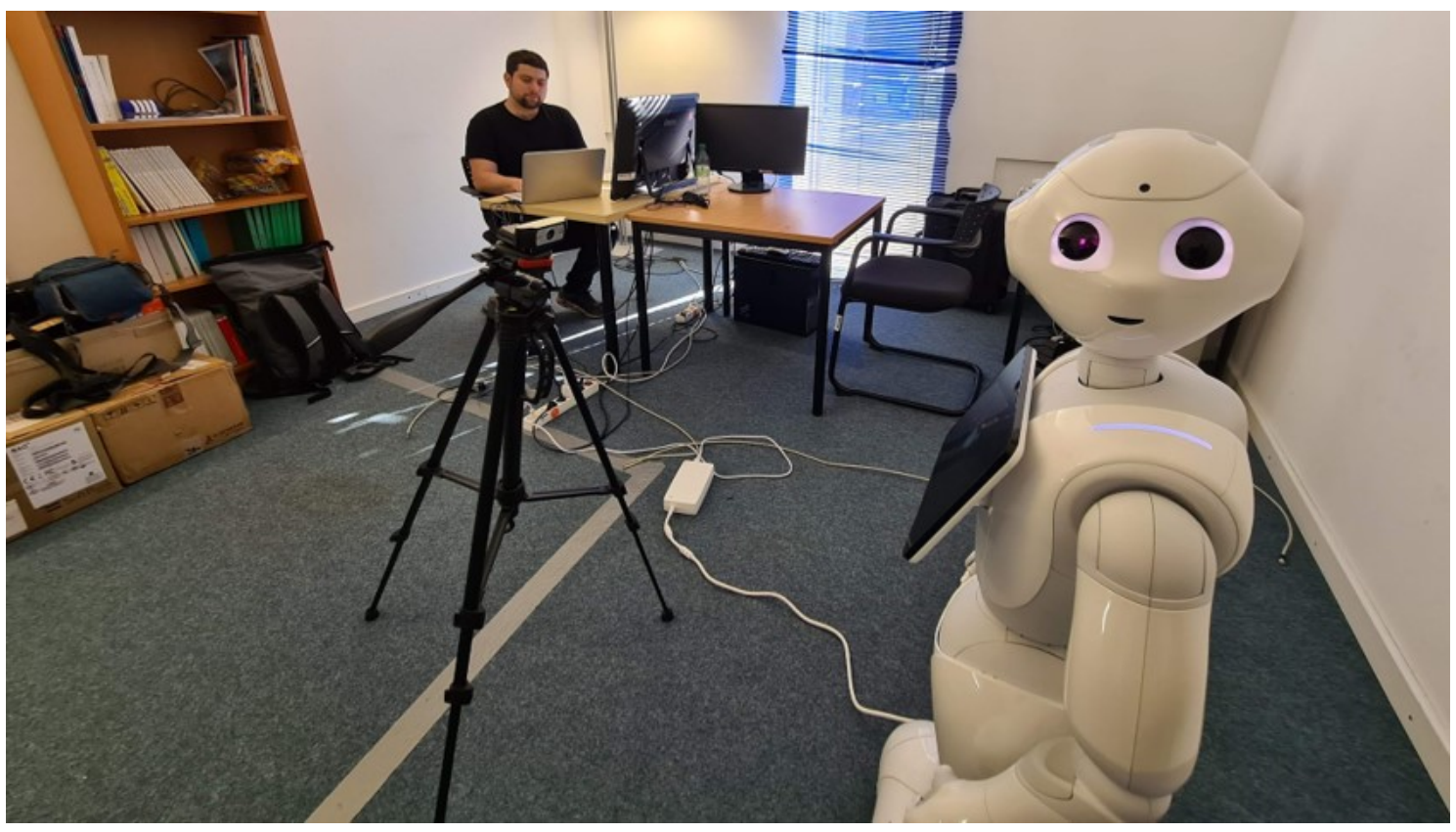

Figure 1: The lab settings, including the robot Pepper (SoftBank Robotics) in front of a web camera, while the experimenter in the back is controlling the robot using the Wizard of $\mathrm{Oz}$ technique.

Conversational interactions were guided by the robot Pepper (SoftBank Robotics), a humanoid robot capable of communicating via speech and body language or gestures. Following (51) guidelines for social robots' design for long-term interactions, Pepper was chosen as a suitable robotic platform for this task, given the alignment between Pepper's humanoid embodiment and the social requirements of the conversational task (see 51, "Guidelines for Future Design"). While Pepper's appearance and behaviours are somewhat human-like (i.e., Pepper has a head, face, torso, two arms, two hands, five fingers per hand, etc.), Pepper has not been designed to resemble a real person. Instead, Pepper's embodiment and behaviours clearly convey human-likeness, (further evidenced, for example, by Pepper's abilities to communicate using human speech, but not demonstrating any facial expressions given the rigid, immobile face and head).

Pepper was placed in front of a web camera (Logi-tech, 1080p), connected to the experimenter computer (see Figure 11. Behind Pepper was a white wall and a flowerpot with a green plant (see Figure 2). Pepper communicated with participants in this study via the WoZ technique controlled by the experimenter via a PC laptop. All pre-scripted 
questions and speech items were written and coded in the WoZ system, with the experimenter (GL) controlling Pepper by pressing buttons on a PC laptop. Accordingly, the procedure followed a clear pre-programmed protocol where the experimenter did not need to speak or type anything during the interaction, but only pressed the relevant keys to trigger the required or appropriate text delivery via Pepper.

Pepper responded to participants' answers and statements with neutral or empathetic responses. Pepper's vocabulary was limited and constrained to reflect the current state of speech recognition technology in social robotics. Following (51) guidelines for social robots' design for long-term interactions, Pepper's responses were affective and empathetic, aiming to convey an understanding of users' affective state, communicate appropriate responses, and also display contextualised affective reactions (see 51, "Guidelines for Future Design"). Hence, a limited set of responses were pre-defined for answers and statements with neutral sentiment or containing factual information (e.g., "I understand", "I see", "okay"), for answers and statements of positive sentiment (e.g., "I am happy to hear that", "This is really interesting", "That's amazing"), and for answers and statements of negative sentiment (e.g., "I am sorry to hear that", "This sounds very challenging", "These are not easy times"). Moreover, Pepper had pre-defined statements for opening an interaction (e.g., "Hello there", "Hi!", "How are you doing today?"), closing an interaction ("That's it for now", "See you next time", "Have a good weekend", "Goodbye"), answer with basic polite gratitude (e.g., "I am fine, thank you!", "Thank you", "That is lovely of you to say so", "It was nice to chat with you too!"), and thank participants for their cooperation and disclosures (e.g., "Thank you for sharing with me", "Thank you for telling me", "What a nice memory. Thank you for sharing with me"). Due to Pepper's high pitched voice and robotic style of pronunciation, Pepper's answers and statements were structured using commas so that Pepper's speech segments will be clearer. See the OSF repository $\left(49[]^{1}\right.$ for a file with all of Pepper's vocabulary and the structure of Pepper's speech segments.

Pepper communicated using a cheerful, high-pitched voice, and expressive and animated body language that corresponded to the spoken content and Pepper's physical capabilities. Pepper's movements were self-initiated based on Pepper's demo software's "animation" function, in order to provide a sense of neutral interaction and to ensure replicability by future studies using the same functionality that all Pepper robots are equipped with. Moreover, Pepper's gaze was almost always focused on the camera, but it shifted and moved from the camera with no pre-programmed logic. To ensure that the mediated interactions would come across as natural, Pepper's gaze was not programmed to be focused on the camera at all times as this would not be normal behaviour with conversing with a human interlocutor. Therefore, Pepper's gaze shifts were allowed to naturally occur following its demo software.

\subsection{Manipulation}

\subsubsection{Structure}

In accordance to (51) guidelines for social robots' design for long-term interactions, the interactions followed a clear structure and routine, including greetings and farewells, identifying participants by their name, and demonstrating appropriate affective and emphatic responses to participants' answers to provide a sense of personal interactions and encourage self disclosure (see 51, "Guidelines for Future Design").

Each interaction with the robot followed the same order, starting with greetings followed by 3 questions (x3 repetitions). The participants were instructed to have a short conversation with the robot, following the robot's lead in the interaction and answering the robot's questions. Participants were instructed that no time limit applied for the interactions and that the interactions usually took about five to ten minutes. They were further encouraged to participate in the interactions the way they saw fit - speaking as little or as much as they wished. In addition, participants were instructed that there were no correct or incorrect answers, and they were encouraged to provide honest answers according to what they feel comfortable with. In the first interaction with the robot (Session 1), participants were their name by the robot as part of the robot introduction (i.e., "Hello there, my name is Pepper, what is your name?), as such a question would be part of a normal introduction in on-going social exchanges with another person. Before the interaction started, participants were

\footnotetext{
${ }^{1}$ https://osf.io/m74cb/
} 
instructed that they were not obliged to share their name with the robot, and that they could give a fake name if they preferred to do so. From the second interaction (Session 2) onwards, the robot addressed each participant by the name they gave during the first interaction (Session 1), to provide a sense of natural and personalized interactions.

Each short interaction was guided by the robot as a semi-structured interview discussing non-sensitive topics regarding general everyday experiences. The task followed the following structure and order:

- Short greetings/introduction (e.g., Hi there, how are you doing?).

- One pre-defined general question about the participant day, week, or weekend, to build rapport (e.g., "how was your weekend? Did you do anything interesting?").

- An opening statement introducing the topic of the question (e.g., "I am about to ask you about your social life").

- Two pre-defined, non-sensitive questions that correspond to the topic that was randomly allocated to the interaction. These questions were either framed in the context of the COVID-19 pandemic or to a more general everyday context, depending on the discussion theme group assignment.

\subsubsection{Content}

Previous studies that investigated relationship formation and disclosure with artificial agents followed conceptual frameworks for inducing rich disclosures and forming meaningful connections (e.g., 19; 18, 48; 62). For example, a study by (18) presented an implementation of 36 questions as a method to generate interpersonal closeness ("36 questions to love"; 3) and elicit self-disclosure from human users to a chatbot. In a previous study (48), we demonstrated how simple questions about everyday experiences (i.e., work-life balance and finances, social life and relationships, and health and well-being) can elicit meaningful disclosures when communicated by a social robot. The questions and topics in the study were influenced by (42) and (41) as an elicitation technique. (41) research was intended to capture one's subjective views regarding their own disclosures, however, it is also introducing everyday topics and matters that people often discuss, sharing non-sensitive information, but that can also touch more personal matters depending on the context of the interaction (see 46). Here we were using a similar type of questions to the ones used in (48), adapting disclosure topics for the ten sessions from (41) and (17), and framing the disclosure topics and questions in this study following a framework and guidelines by (17).

\begin{tabular}{|c|c|c|}
\hline & Topic & Category \\
\hline 1 & Work & Control, Autonomy, and Choice \\
\hline 2 & Leisure and Passions (Life) & All \\
\hline 3 & Finances & Control, Autonomy, and Choice \\
\hline 4 & Relationships & Belonging \\
\hline 5 & Social life & Belonging \\
\hline 6 & Mental Health & Well-being and Ill-being \\
\hline 7 & Physical Health & Well-being and Ill-being \\
\hline 8 & Personality & Self-Perception \\
\hline 9 & Goals and Ambitions & Hope and Hopelessness \\
\hline 10 & Routine and Daily Activities & Activity \\
\hline
\end{tabular}

Table 1: The ten topics and corresponding quality of life categories following (17) framework.

The framework by (17) introduces guidelines via six main themes for asking questions that capture and elicit disclosures that relate to different elements of quality of life within counselling psychology settings and mental health therapy. The guidelines and themes were defined by (17) after reviewing and synthesizing qualitative research studies (especially from the counselling psychology literature, psychotherapy, and mental health therapy literature) that explicitly asked adult participants with mental health problems about the factors they considered important to their quality of life or how it had been impacted by their mental health. Based on (17) review's results the six themes are: (1) Well-being 
and Ill-being, (2) Control, Autonomy, and Choice, (3) Self-Perception, (4) Belonging, (5) Activity, (6) Hope and Hopelessness.

The ten topics for the ten sessions describe one or more of the six themes described by (17), aiming to elicit meaningful disclosures following (41) guidelines, but also to initiate self-reflection and capture meaningful information regarding the quality of life and mental health, following (17) framework. The ten topics and their corresponding themes according to (17) framework can be seen in table1. The phrasing of each of the two questions under each topic followed (3) approach for questions and practical methodology for creating interpersonal closeness in experimental context (see the questions in table 21).

\subsubsection{Discussion Themes}

For both discussion themes, the interaction always started the same way, with greetings and with the robot asking the first question about the participant's day/week/weekend (see section2.4.1 for the structure of the task). The following two pre-defined questions were about a topic that was randomly allocated to the interaction from that the 10 topics about general everyday experiences (see table 1 ).

For participants assigned to the neutral discussion theme group, the questions were not limited to any specific frame other than general everyday context (see table 2).

For participants assigned to the Covid-related discussion theme group, questions were asked about the same topics, however, the questions were framed within the context of the COVID-19 pandemic. For example, participants were asked how their work situation changed due to the pandemic, or how they were socializing during the pandemic (see the questions and differences between conditions in table 2 .

\subsection{Procedure}

\subsubsection{Zoom video chat}

All the interactions (video chats) were conducted with the software Zoom, using a University of Glasgow staff account. The interactions were recorded using the recording functionality on Zoom and edited to include only those portions of the recordings where participants and/or Pepper are speaking.

\subsubsection{Qualtrics form}

All of the questionnaires were administered via the survey software Qualtrics, using a University of Glasgow staff account. In the online questionnaires, the functionality recording participants' IP addresses were disabled in order to comply with GDPR guidelines.

\subsubsection{Session 0 - Induction Questionnaire}

When recruited, participants completed an induction questionnaire (Session 0) approximately one week before beginning their video chat interactions with Pepper (Sessions 1 to 10). Participants were instructed to have a short conversation with the robot about several topics that the robot will bring up, that the robot will ask them 3 questions and that the interactions will take place twice a week across five weeks during prearranged times. They were further told that each interaction with the robot should last about 5 to 10 minutes, and another 10-15 minutes will be required in order to complete questionnaires afterwards. See section 2.2 .3 for more information about the recruitment procedure, and section 2.2.4 for ethics consideration and communications with the participants.

When answering the induction questionnaire (after providing consent to participate in the study), participants were instructed on how to position their video camera for their interactions with the robot, and how the lighting in the room is expected to be like. Following this, participants reported on several demographic parameters, including: 


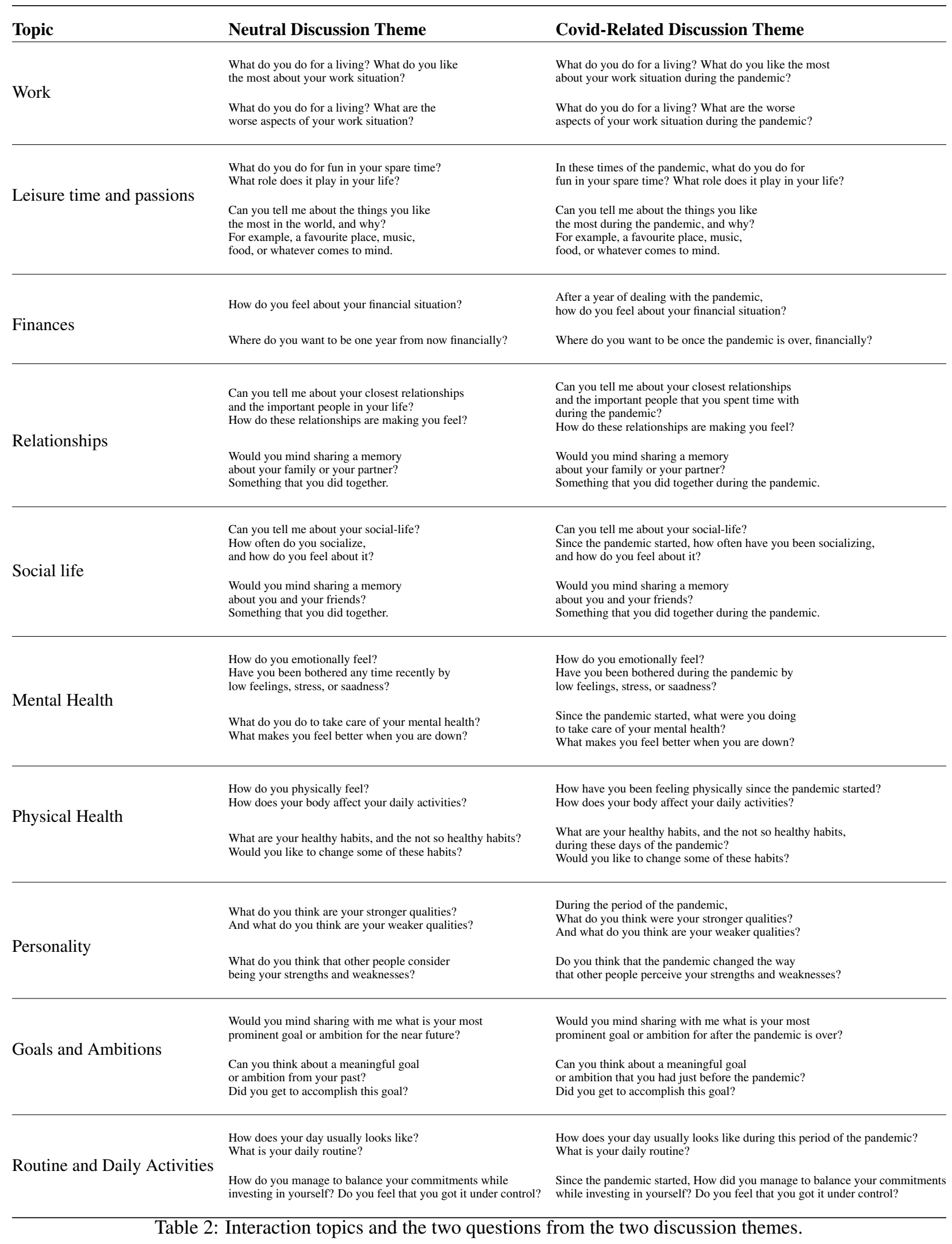


• First language (i.e., "Is English your first language?").

- Age in years.

- Gender identity.

- Biological sex.

- Highest education level achieved.

- Employment status.

- Occupation sector.

- Martial status.

- Number of people living at the same house.

- Country of origin.

- Country of residence.

- Previous experience with social robots.

Then, participants filled these questionnaires in the following order:

- Distress disclosure index (43, 44).

- Perceived stress (PPS; 16).

- the short-form UCLA loneliness scale (ULS-8; 31).

- COVID-19 threat perception (38).

- COVID 19 worries scale (An adapted and shorter version of "COVID Stress Scales" by (author?) (74)).

- COVID-19 conspiracy belief scale (38).

- Extraversion items of the Mini-Markers Big Five personality scale (66).

When completing the induction questionnaire (Session 0), participants were redirected to the Prolific website. A participant number was automatically generated for each participant (1 to 40) who completed the induction questionnaire (Session 0) and proceeded to the following sessions. The random assignment of participants to conditions, allocation of topics to sessions for each participant and the order of questions in each interaction were randomized using a simple python script (see python notebook on OSF repository at 49 . An excel sheet was created to help the experimenter control and follow the interactions and sessions when running the experiment for five weeks (see the experimenter notebook with the conditions, allocated topics to a session, and order of questions for each of the participants on the OSF repository at 49 ).

\subsubsection{Sessions 1 to 10}

Participants were reminded about each session before it took place (see section 2.2.4 and could find the link for each session on the Prolific study page of the session, which became available on the morning of the session.

Before starting each session, participants were instructed to wait to their individual time slot so that they will not disrupt other participants' sessions. They were informed and reminded about the session number and were encouraged to switch off their phone/e-mail/music, and other potential distractions so that they could focus on their participation in the study. They were also reminded that they can only participate using a desktop or notebook computer, and not using smartphones and tablets. When starting each session, participants were asked to enter their Prolific ID and their participant number. Following, participants were asked to answer the Immediate Mood Scaler (IMS-12; 58) for

\footnotetext{
${ }^{1}$ https://osf.io/m74cb/
} 


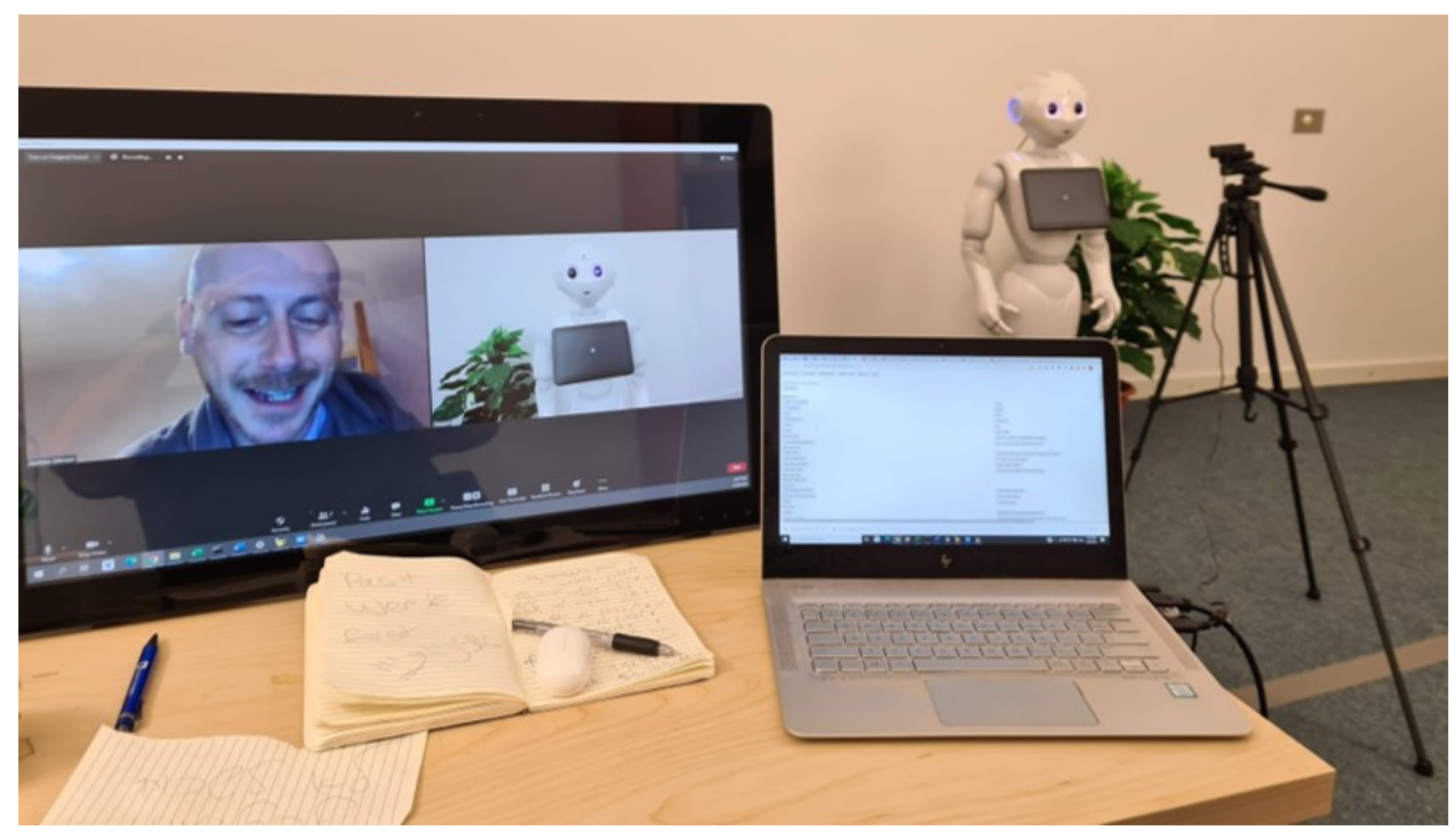

Figure 2: The interaction from the eyes of the participants and the experimenter. The participants were exposed only to the robot Pepper (SoftBank Robotics) via the zoom chats.

reporting their mood before interacting with Pepper. Next, participants received a reminder regarding their interaction with Pepper and what the task requires and some basic instructions (see Figure 3). The page included a link to the zoom interaction, a frame with the zoom landing page, and the experimenter (GL) e-mail address and instructions on how to communicate with the experimenter in case there are any issues during the interaction (see Figure 3 ).

After finishing their interaction with Pepper, participants went back to the Qualtrics page and answered the rest of the questionnaires in the following order:

- Agency and Experience (adapted from 30).

- Comforting responses scale (adapted from 15 ).

- Subjective self-disclosure (an adaptation of the work and studies sub-scale from Jourard's Self-Disclosure questionnaire (41)).

- Post interaction IMS-12 (adapted from 58).

Then, participants evaluated the robot and the interaction on several scales, including:

- Interaction quality (an adapted and adjusted version by (19) for a scale by (8)). Each interaction included two random items out of six, except for the mid-session (session 5) and the last session (session 10) that included all six items of the scale.

- Communication competence (an adapted and adjusted version by (19) for a scale by (24)).

- Disclosure quality (an adapted and adjusted version by (19) for a scale by (50)).

- Friendliness and warmth (one item from (61) and two items from (9), as suggested by (35)).

Finally, participants reported their feelings of loneliness via the ULS-8 (31). When finishing answering the questionnaire, participants were thanked for the completion of the session, reminded about the date and day of their upcoming session, were provided again with the contact details of the experimenter (GL), and were directed back to Prolific to receive a completion message. 
You are about to start your interaction with Pepper. In the following interaction Pepper is going to be asking you three different questions -

all you are required to do is answering these questions.

There are no correct or incorrect answers, you are encouraged to provide honest answers. Please follow Pepper's guidance and instructions, and do not interrupt Pepper.

Please wait in this page until finishing the interaction with Pepper. Continue with the survey only once the interaction on Zoom ends and Pepper tells you that you finished and that you may continue.

To start the interaction using Zoom - PRESS HERE

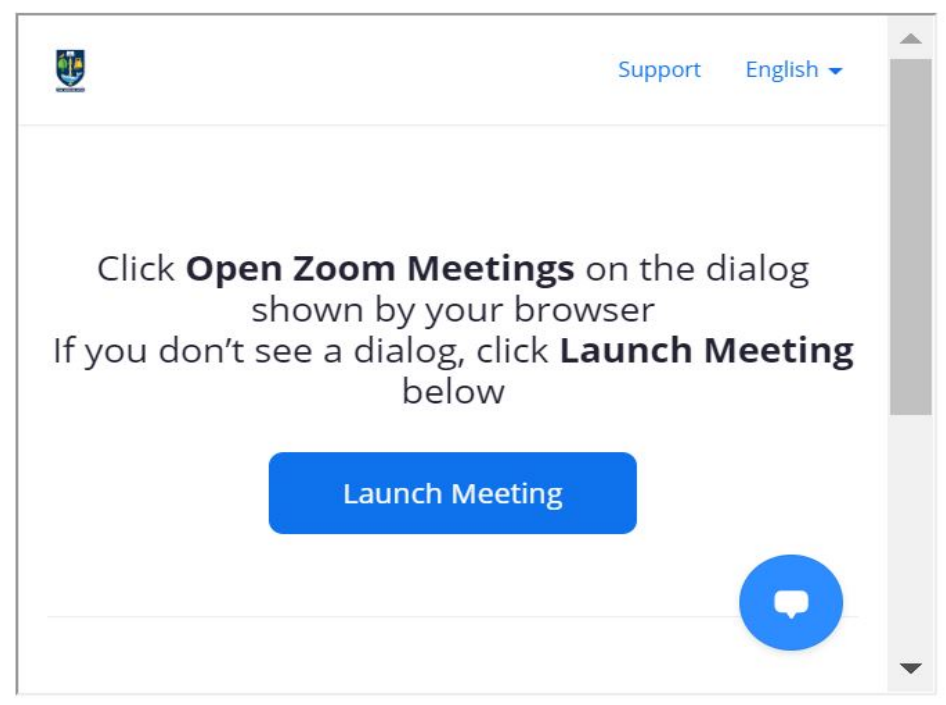

If there are any issues during the interaction, please feel free to bring them to the experimenter attention at any time using the Prolific messaging system or by email at Guy.Laban@glasgow.ac.uk.

Figure 3: The interaction page leading to the Zoom video chat in the Qualtrics form

\subsubsection{Unique Sessions}

Session 3 In session 3, before completing the session participants were asked if they could answer three more additional open-ended questions. The participants were clarified that this part is optional and not obligatory. The open-ended questions were:

- What do you think about Pepper at this point of your participation?

- What are the best things about talking to Pepper?

- What are the worse things about talking to Pepper? 
Session 5 - Mid-Session In the Mid-session (session 5), participants followed the same procedure as in the other sessions, but also answered to the following additional questionnaires:

- Interaction quality (an adapted and adjusted version by (19) for a scale by $(\underline{8})$ ). Each interaction included two random items out of six, except for the mid-session (session 5) and the last session (session 10) that included all six items of the scale.

- PPS (16).

- COVID-19 threat perception (38).

Session 10 - Last Session In the last session (session 10), participants followed the same procedure as in the other sessions, but also answered the following additional questionnaires:

- Interaction quality (an adapted and adjusted version by (19) for a scale by (8)). Each interaction included two random items out of six, except for the mid-session (session 5) and the last session (session 10) that included all six items of the scale.

- Participants were asked if they are going to miss Pepper (a yes or no question).

- Distress disclosure index (43, 44).

- PPS (16).

- COVID-19 threat perception (38).

- COVID 19 worries scale (An adapted and shorter version of "COVID Stress Scales" by (74)).

- COVID-19 conspiracy belief scale (38).

Then, participants were asked the following open-ended questions:

- What do you think about Pepper after your participation in the study?

- What are the best things about talking to Pepper?

- What are the worse things about talking to Pepper?

- Can you please tell us about your relationship with Pepper? How did Pepper make you feel in the past month?

- Can you please share a prominent memory that you have from your interactions with Pepper?

Finally, participants reported on the following items concerning their quality of life during the COVID-19 pandemic.

- Social isolation and social participation (an adjusted version of the social participation questionnaire (25) and the short social participation questionnaire for lockdowns $(2,1 ; 4)$ ).

- Life satisfaction - before and during the pandemic (an adjusted version of the the short life satisfaction questionnaire for lockdowns (1; 4) that was influenced from (26)).

Before completing the session participants were asked to rate their communication with the experimenter (GL), and if they could answer two short additional open-ended questions. The participants were clarified that this part is optional and not obligatory. In this part the participants were asked what do they think that this study was testing, and if they have any comments or feedback about the study. When completing the last session participants were clarified that this is indeed the last session, they were thanked for their participation, provided with contact details of the experimenter (GL) to ask any further questions about the study. Moreover, participants were clarified that as long as they participated in all sessions they will be granted the bonus payment of $£ 20$ as soon as possible. 


\section{Discussion}

Here we have introduced a novel long-term mediated experimental protocol aimed to test the extent to which social robots can be used to elicit emotionally-rich disclosures and expressions from people in their own homes, across time, in order to further our understanding of how robots might be used to help community and health professionals identify people's needs and emotional states over time. The present protocol aims to establish experimental methods that researchers from a number of different fields, including psychology, psychiatry, social work, and computer science might wish to use to further explore people's perceptions of a sociable, humanoid robot in natural everyday settings during prolonged conversational interactions. As well as exploring general questions regarding how people engage with a social robot from their home settings, the current protocol also provides a means to further examine the impact of novelty effects, and the impact of long-term social engagement with a robot on behaviour (c.f., 21). By introducing this novel protocol in detail here, our aim is to provide a tool that will hopefully be of use to the HRI research community more broadly, which will also assist with facilitating research rigour and reproducibility $(33,20)$. Moreover, we would argue that the online computer mediated means of human-robot communication used in this experimental design can overcome some of the challenges and barriers that are related to long-term HRI studies in natural ecologically valid settings (such as the costs associated with sending individual robots home for an extended period of time with participants).

The effective delivery of long-term user studies has long been a goal (and a challenge) for HRI and social robotics researchers (c.f., $7,11,21 ;, 22,23,29 ; 51,53,71 ; 73$ ). Of the handful of long-term user studies in real-world environments that have been run (e.g., $11,21,22,23 ; 29,52,54)$, these are rarely replicated to demonstrate robustness of results in a systematic fashion (see 6; 33). Accordingly, while longitudinal effects of repeated HRIs are being increasingly explored in the HRI research community (for example, in the recent Frontiers Research Topics on Novelty effects in HRI ${ }^{2}$ and Lifelong Learning and Long-Term HRIs 3 , such long term engagement studies, and the findings they produce, still require careful validation and replication (5). This will ensure the burgeoning fields of HRI and social robotics can sidestep the deeply damaging impacts that the well-publicised replication crises in psychology and neuroscience have wrought Irfan et al. (39). As a number of others have recently argued (20, 39; 33, 34), it will be beneficial if HRI researchers can develop novel methodologies that take into account best practices from across the engineering and the social sciences (specifically social and cognitive psychology), while learning from the mistakes and successes of these disciplines. Here we have attempted to take steps toward this call to action through presentation of a novel research paradigm designed to address major theoretical and practical challenges in the field (e.g., long-term HRI, the role of novelty in HRI, as well as how these interactions take place in natural ecologically valid settings, and a clear pathway for replication).

Due to the constrained and highly choreographed nature of much of HRI research, deep insights into people's responses and interactions with robots in natural settings remain relatively rare. Of the field studies that have conducted HRI research in these spaces, important insights are emerging from single interaction (e.g., 72, 36) and repeated interaction (e.g., 11; 22, 23; 29, 52; 54) studies, with much of this work taking place in public spaces or tied to specific settings like education (e.g., 45, 52, 55; 56, 75), care (e.g., 63, 64, 60, 54), or rehabilitation (e.g., 11, 12, 29). Longitudinal studies that addresses similar questions with disembodied agents such as virtual assistants and chatbots (e.g., 18, 19; 35) benefit from access to users' personal devices, whereas research with physically embodied artificial agents (i.e., social robots) remains far rarer due to challenges with logistical and cost barriers to situating these devices in users' domestic settings (i.e., in their home environment) to explore single or repeated interactions. While several attempts have been made before to reduce the barriers to feasibility for such work (c.f., 13, 21; 22, 23), we still know relatively little about user perceptions of and behaviours toward social robots when these take place in familiar home environments. Further insights into the challenges and opportunities afforded by placing social robots into familiar domestic settings should

\footnotetext{
${ }^{2}$ https://www.frontiersin.org/research-topics/16567/novelty-effects-in-human-robot-interaction

${ }^{3}$ https://www.frontiersin.org/research-topics/14495/lifelong-learning-and-long-term-human-robot-interaction
} 
aid human-robot communication in general, as well as further refine the development and utility of these machines for commercial use.

Social robots hold great potential for delivering or improving psycho-social interventions (65), supporting mental health (68), monitoring symptoms of chronic pathologies (47), and providing much-needed physical and social support across a number of daily life settings (34). The recent COVID-19 pandemic further illuminated the potential of social robots as an assistive technology in times when strict infection control measures mandate physical distancing between people. A number of researchers have argued that physically embodied social robots should be able to assist with a number of tasks to help keep people physically and mentally healthy, ranging from temperature taking and food and supply delivery to providing companionship for individuals suffering from loneliness $(32,34,67,76)$, and even mediating social interactions with other individuals (40). Nevertheless, as discussions concerning the potential applications for social robots became more prominent during the pandemic, HRI research was limited due to social distancing and the inability to use lab facilities for research that is highly dependent on laboratory constrained environments. The pandemic forced most individuals (including researchers) to adopt computer-mediated means of communication (CMC) (14). Following the wholesale adaptation to $\mathrm{CMC}$ during the pandemic, the current protocol sets forth a means for conducting rigorous and reproducible social robotics research to explore people's engagement with social robot-mediated interactions within their own homes. More generally, this protocol sets the stage for further research exploring online mediated speech-based psychosocial interventions with social robots when public health, cost, or logistical barriers prevent situating a physically embodied robot in users homes across the long term.

\section{Acknowledgements}

The authors gratefully acknowledge funding from the European Union's Horizon 2020 research and innovation programme under the Marie Sklodowska Curie to ENTWINE, the European Training Network on Informal Care (grant agreement no. 814072), the European Research Council (ERC) under the European Union's Horizon 2020 research and innovation programme (Grant agreement 677270 to E.S.C.), and the Leverhulme Trust (PLP-2018-152 to E.S.C.).

\section{References}

[1] Ammar, A., Chtourou, H., Boukhris, O., Trabelsi, K., Masmoudi, L., Brach, M., Bouaziz, B., Bentlage, E., How, D., Ahmed, M., Mueller, P., Mueller, N., Hsouna, H., Aloui, A., Hammouda, O., Paineiras-Domingos, L.L., Braakman-Jansen, A., Wrede, C., Bastoni, S., Pernambuco, C.S., Mataruna, L., Taheri, M., Irandoust, K., Khacharem, A., Bragazzi, N.L., Strahler, J., Adrian, J., Andreeva, A., Khoshnami, S.C., Samara, E., Zisi, V., Sankar, P., Ahmed, W.N., Romdhani, M., Delhey, J., Bailey, S.J., Bott, N.T., Gargouri, F., Chaari, L., Batatia, H., Ali, G.M., Abdelkarim, O., Jarraya, M., Abed, K.E., Souissi, N., Van Gemert-Pijnen, L., Riemann, B.L., Riemann, L., Moalla, W., Gómez-Raja, J., Epstein, M., Sanderman, R., Schulz, S., Jerg, A., Al-Horani, R., Mansi, T., Jmail, M., Barbosa, F., Santos, F., Šimunič, B., Pišot, R., Pišot, S., Gaggioli, A., Zmijewski, P., Apfelbacher, C., Steinacker, J., Saad, H.B., Glenn, J.M., Chamari, K., Driss, T., Hoekelmann, A.: Covid-19 home confinement negatively impacts social participation and life satisfaction: A worldwide multicenter study. International Journal of Environmental Research and Public Health 17(17), 1-17 (2020). doi:10.3390/ijerph17176237. URL wWw.mdpi.com/journal/ijerph

[2] Ammar, A., Trabelsi, K., Brach, M., Chtourou, H., Boukhris, O., Masmoudi, L., Bouaziz, B., Bentlage, E., How, D., Ahmed, M., Mueller, P., Mueller, N., Hammouda, O., Paineiras-Domingos, L.L., Braakman-Jansen, A., Wrede, C., Bastoni, S., Pernambuco, C.S., Mataruna, L., Taheri, M., Irandoust, K., Khacharem, A., Bragazzi, N.L., Strahler, J., Adrian, J., Andreeva, A., Glenn, J.M., Bott, N.T., Gargouri, F., Chaari, L., Batatia, H., Khoshnami, S.C., Samara, E., Zisi, V., Sankar, P., Ahmed, W.N., Ali, G.M., Abdelkarim, O., Jarraya, M., Abed, K.E., Moalla, W., Romdhani, M., Aloui, A., Souissi, N., van Gemert-Pijnen, L., Riemann, B.L., Riemann, L., Delhey, J., Gómez-Raja, J., Epstein, M., Sanderman, R., Schulz, S., Jerg, A., Al-Horani, R., Mansi, T., Jmail, M., Barbosa, 
F., Ferreira-Santos, F., Šimunič, B., Pišot, R., Pišot, S., Gaggioli, A., Zmijewski, P., Bailey, S.J., Steinacker, J., Chamari, K., Driss, T., Hoekelmann, A.: Effects of home confinement on mental health and lifestyle behaviours during the COVID-19 outbreak: Insights from the ECLB-COVID19 multicentre study. Biology of Sport 38(1), 9-21 (2021). doi:10.5114/biolsport.2020.96857 URL/pmc/articles/PMC7996377//pmc/articles/ PMC7996377/?report=abstracthttps://www.ncbi.nlm.nih.gov/pmc/articles/PMC7996377/

[3] Aron, A., Melinat, E., Aron, E.N., Vallone, R.D., Bator, R.J.: The experimental generation of interpersonal closeness: A procedure and some preliminary findings. Personality and Social Psychology Bulletin 23(4), 363-377 (1997). doi:10.1177/0146167297234003. URL https://journals.sagepub.com/doi/10.1177/ 0146167297234003

[4] Bastoni, S., Wrede, C., Ammar, A., Braakman-Jansen, A., Sanderman, R., Gaggioli, A., Trabelsi, K., Masmoudi, L., Boukhris, O., Glenn, J.M., Bouaziz, B., Chtourou, H., van Gemert-Pijnen, L.: Psychosocial effects and use of communication technologies during home confinement in the first wave of the covid-19 pandemic in Italy and the Netherlands. International Journal of Environmental Research and Public Health 18(5), 1-12 (2021). doi:10.3390/ijerph18052619. URL https://www.mdpi.com/1660-4601/18/5/2619/htmhttps: //www.mdpi.com/1660-4601/18/5/2619

[5] Belpaeme, T.: Advice to New Human-Robot Interaction Researchers pp. 355-369 (2020). doi:10.1007/978-3-03042307-0_14. URL https://link.springer.com/chapter/10.1007/978-3-030-42307-0_14

[6] Belpaeme, T.: Learning from Social Robots. In: 2020 International Symposium on Community-centric Systems (CcS), p. 12 (2020). doi $10.1109 / \mathrm{CcS} 49175.2020 .9231310$

[7] Ben-Youssef, A., Clavel, C., Essid, S., Bilac, M., Chamoux, M., Lim, A.: UE-HRI: A new dataset for the study of user engagement in spontaneous human-robot interactions. ICMI 2017 - Proceedings of the 19th ACM International Conference on Multimodal Interaction 2017-January, 464-472 (2017). doi:10.1145/3136755.3136814

[8] Berry, D.S., Hansen, J.S.: Personality, Nonverbal Behavior, and Interaction Quality in Female Dyads. Personality and Social Psychology Bulletin 26(3), 278-292 (2000). doi 10.1177/0146167200265002. URL https://doi. org/10.1177/0146167200265002

[9] Birnbaum, G.E., Mizrahi, M., Hoffman, G., Reis, H.T., Finkel, E.J., Sass, O.: What robots can teach us about intimacy: The reassuring effects of robot responsiveness to human disclosure. Computers in Human Behavior 63, 416-423 (2016). doi:10.1016/j.chb.2016.05.064

[10] Breazeal, C.: Toward sociable robots. Robotics and Autonomous Systems 42(3), 167-175 (2003). doi:10.1016/S0921-8890(02)00373-1

[11] Céspedes, N., Irfan, B., Senft, E., Cifuentes, C.A., Gutierrez, L.F., Rincon-Roncancio, M., Belpaeme, T., Múnera, M.: A Socially Assistive Robot for Long-Term Cardiac Rehabilitation in the Real World. Frontiers in Neurorobotics 15, 21 (2021). doi 10.3389/FNBOT.2021.633248 URL www.frontiersin.org

[12] Céspedes, N., Raigoso, D., Múnera, M., Cifuentes, C.A.: Long-Term Social Human-Robot Interaction for Neurorehabilitation: Robots as a Tool to Support Gait Therapy in the Pandemic. Frontiers in Neurorobotics 15, 10 (2021). doi:10.3389/FNBOT.2021.612034. URL www.frontiersin.org

[13] Chaudhury, B., Hortensius, R., Hoffmann, M., Cross, E.S.: Tracking Human Interactions with a Commerciallyavailable Robot over Multiple Days : A Tutorial. PsyArXiv (2020). doi:10.31234/OSF.IO/FD3H2. URL https://psyarxiv.com/fd3h2/

[14] Choi, M., Choung, H.: Mediated communication matters during the COVID-19 pandemic: The use of interpersonal and masspersonal media and psychological well-being:. https://doi.org/10.1177/02654075211029378 p. 026540752110293 (2021). doi $10.1177 / 02654075211029378$. URL https://journals.sagepub.com/doi/ abs/10.1177/02654075211029378 
[15] Clark, R.A., Pierce, A.J., Finn, K., Hsu, K., Toosley, A., Williams, L.: The impact of alternative approaches to comforting, closeness of relationship, and gender on multiple measures of effectiveness. Communication Studies 49(3), 224-239 (1998). doi 10.1080/10510979809368533 URL https://doi.org/10.1080/10510979809368533

[16] Cohen, S., Kamarck, T., Mermelstein, R.: A global measure of perceived stress. Journal of health and social behavior 24(4), 385-396 (1983). doi:10.2307/2136404

[17] Connell, J., Brazier, J., O'Cathain, A., Lloyd-Jones, M., Paisley, S.: Quality of life of people with mental health problems: a synthesis of qualitative research. Health and Quality of Life Outcomes 10(1), 138 (2012). doi:10.1186/1477-7525-10-138. URL https://doi.org/10.1186/1477-7525-10-138

[18] Croes, E.A., Antheunis, M.L.: 36 Questions to Loving a Chatbot: Are People Willing to Self-disclose to a Chatbot? In: Lecture Notes in Computer Science (including subseries Lecture Notes in Artificial Intelligence and Lecture Notes in Bioinformatics), vol. 12604 LNCS, pp. 81-95. Springer Science and Business Media Deutschland GmbH (2021). doi:10.1007/978-3-030-68288-0_6 URL https://doi.org/10.1007/978-3-030-68288-0_6

[19] Croes, E.A.J., Antheunis, M.L.: Can we be friends with Mitsuku? A longitudinal study on the process of relationship formation between humans and a social chatbot. Journal of Social and Personal Relationships 38(1), 279-300 (2020). doi:10.1177/0265407520959463. URL https://doi.org/10.1177/0265407520959463

[20] Cross, E.S., Ramsey, R.: Mind Meets Machine: Towards a Cognitive Science of Human-Machine Interactions. Trends in Cognitive Sciences 25(3), 200-212 (2021). doi 10.1016/J.TICS.2020.11.009

[21] Cross, E.S., Riddoch, K.A., Pratts, J., Titone, S., Chaudhury, B., Hortensius, R.: A neurocognitive investigation of the impact of socializing with a robot on empathy for pain. Philosophical Transactions of the Royal Society B: Biological Sciences 374(1771), 20180034 (2019). doi:10.1098/rstb.2018.0034. URL https://royalsocietypublishing.org/doi/abs/10.1098/rstb.2018.0034

[22] De Graaf, M.M., Allouch, S.B., Klamer, T.: Sharing a life with Harvey: Exploring the acceptance of and relationship-building with a social robot. Computers in Human Behavior 43, 1-14 (2015). doi:10.1016/J.CHB.2014.10.030

[23] De Graaf, M.M.A., Allouch, S.B., van Dijk, J.A.G.M.: Long-term evaluation of a social robot in real homes. Interaction studies 17(3), 462-491 (2016)

[24] Demeure, V., Niewiadomski, R., Pelachaud, C.: How Is Believability of a Virtual Agent Related to Warmth, Competence, Personification, and Embodiment? Presence: Teleoperators and Virtual Environments 20(5), 431-448 (2011). doi:10.1162/PRES_a_00065. URL https://doi.org/10.1162/PRES_a_00065

[25] Densley, K., Davidson, S., Gunn, J.M.: Evaluation of the Social Participation Questionnaire in adult patients with depressive symptoms using Rasch analysis. Quality of Life Research 22(8), 1987-1997 (2013). doi:10.1007/s11136-013-0354-4. URL https://pubmed.ncbi.nlm.nih.gov/23341174/

[26] Diener, E., Emmons, R.A., Larsem, R.J., Griffin, S.: The Satisfaction With Life Scale. Journal of Personality Assessment 49(1), 71-75 (1985). doi:10.1207/s15327752jpa4901_13. URL https://pubmed.ncbi.nlm.nih. gov/16367493/

[27] Faul, F., Erdfelder, E., Buchner, A., Lang, A.G.: Statistical power analyses using G*Power 3.1: Tests for correlation and regression analyses. Behavior Research Methods 41(4), 1149-1160 (2009). doi 10.3758/BRM.41.4.1149. URL https://doi.org/10.3758/BRM.41.4.1149

[28] Faul, F., Erdfelder, E., Lang, A.G., Buchner, A.: G*Power 3: A flexible statistical power analysis program for the social, behavioral, and biomedical sciences. Behavior Research Methods 39(2), 175-191 (2007). doi:10.3758/BF03193146 URL https://doi.org/10.3758/BF03193146

[29] Feingold Polak, R., Tzedek, S.L.: Social Robot for Rehabilitation: Expert Clinicians and Post-Stroke Patients' Evaluation Following a Long-Term Intervention. In: Proceedings of the 2020 ACM/IEEE International Conference 
on Human-Robot Interaction, HRI '20, p. 151-160. Association for Computing Machinery, New York, NY, USA (2020). doi:10.1145/3319502.3374797, URL https://doi.org/10.1145/3319502.3374797

[30] Gray, H.M., Gray, K., Wegner, D.M.: Dimensions of Mind Perception. Science 315(5812), 619 LP - 619 (2007). doi:10.1126/science.1134475. URL http://science.sciencemag.org/content/315/5812/619. abstract

[31] Hays, R.D., DiMatteo, M.R.: A Short-Form Measure of Loneliness. Journal of Personality Assessment 51(1), 6981 (1987). doi:10.1207/s15327752jpa5101_6. URL https://www.tandfonline.com/doi/abs/10.1207/ s15327752jpa5101_6

[32] Henschel, A., Cross, E.S.: The Neuroscience Of Loneliness - And How Technology Is Helping Us. The Conversation pp. 3-7 (2020). URL https://theconversation.com/ the-neuroscience-of-loneliness-and-how-technology-is-helping-us-136093

[33] Henschel, A., Hortensius, R., Cross, E.S.: Social Cognition in the Age of Human-Robot Interaction. Trends in Neurosciences 43(6), 373-384 (2020). doi:https://doi.org/10.1016/j.tins.2020.03.013. URL http://www. sciencedirect.com/science/article/pii/S0166223620300734

[34] Henschel, A., Laban, G., Cross, E.S.: What Makes a Robot Social? A Review of Social Robots from Science Fiction to a Home or Hospital Near You. Current Robotics Reports (2), 9-19 (2021). doi:10.1007/s43154-02000035-0. URL https://doi .org/10.1007/s43154-020-00035-0

[35] Ho, A., Hancock, J., Miner, A.S.: Psychological, Relational, and Emotional Effects of Self-Disclosure After Conversations With a Chatbot. The Journal of communication 68(4), 712-733 (2018). doi $10.1093 /$ joc/jqy026

[36] Holthaus, P., Wachsmuth, S.: It was a Pleasure Meeting You: Towards a Holistic Model of Human-Robot Encounters. International Journal of Social Robotics pp. 1-17 (2021). doi:10.1007/s12369-021-00759-9. URL https://link.springer.com/article/10.1007/s12369-021-00759-9

[37] Hortensius, R., Hekele, F., Cross, E.S.: The Perception of Emotion in Artificial Agents. IEEE Transactions on Cognitive and Developmental Systems 10(4), 852-864 (2018). doi:10.1109/TCDS.2018.2826921

[38] Imhoff, R., Lamberty, P.: A Bioweapon or a Hoax? The Link Between Distinct Conspiracy Beliefs About the Coronavirus Disease (COVID-19) Outbreak and Pandemic Behavior. Social Psychological and Personality Science 11(8), 1110-1118 (2020). doi:10.1177/1948550620934692. URL https://doi.org/10.1177/ 1948550620934692

[39] Irfan, B., Kennedy, J., Lemaignan, S., Papadopoulos, F., Senft, E., Belpaeme, T.: Social Psychology and HumanRobot Interaction: An Uneasy Marriage. In: Companion of the 2018 ACM/IEEE International Conference on Human-Robot Interaction, HRI '18, p. 13-20. Association for Computing Machinery, New York, NY, USA (2018). doi: $10.1145 / 3173386.3173389$. URL https://doi.org/10.1145/3173386.3173389

[40] Isabet, B., Pino, M., Lewis, M., Benveniste, S., Rigaud, A.S.: Social Telepresence Robots: A Narrative Review of Experiments Involving Older Adults before and during the COVID-19 Pandemic. International Journal of Environmental Research and Public Health 18(7) (2021). doi:10.3390/IJERPH18073597. URL /pmc/articles/PMC8037050//pmc/articles/PMC8037050/?report=abstracthttps: //www.ncbi.nlm.nih.gov/pmc/articles/PMC8037050/

[41] Jourard, S.M.: Self-disclosure: An experimental analysis of the transparent self. John Wiley, Oxford, England (1971)

[42] Jourard, S.M., Lasakow, P.: Some factors in self-disclosure. The Journal of Abnormal and Social Psychology 56(1), 91-98 (1958). doi:10.1037/h0043357

[43] Kahn, J.H., Hessling, R.M.: Measuring the Tendency to Conceal Versus Disclose Psychological Distress. Journal of Social and Clinical Psychology 20(1), 41-65 (2001). doi:10.1521/jscp.20.1.41.22254. URL https://doi. org/10.1521/jscp.20.1.41.22254 
[44] Kahn, J.H., Hucke, B.E., Bradley, A.M., Glinski, A.J., Malak, B.L.: The Distress Disclosure Index: A research review and multitrait-multimethod examination. Journal of Counseling Psychology 59(1), 134-149 (2012). doi:10.1037/a0025716

[45] Kanda, T., Hirano, T., Eaton, D., Ishiguro, H.: Interactive robots as social partners and peer tutors for children: A field trial. Human-computer interaction 19(1), 61-84 (2004)

[46] Kreiner, H., Levi-Belz, Y.: Self-Disclosure Here and Now: Combining Retrospective Perceived Assessment With Dynamic Behavioral Measures. Frontiers in Psychology 10, 558 (2019). doi 10.3389/fpsyg.2019.00558. URL https://www.frontiersin.org/article/10.3389/fpsyg.2019.00558

[47] Laban, G., Ben-Zion, Z., Cross, E.S.: Social Robots for Supporting Post-Traumatic Stress Disorder (PTSD) Diagnosis and Treatment (Under Review)

[48] Laban, G., George, J.N., Morrison, V., Cross, E.S.: Tell me more! Assessing interactions with social robots from speech. Paladyn, Journal of Behavioral Robotics 12(1), 136-159 (2021). doi:10.1515/pjbr-2021-0011. URL https://doi.org/10.1515/pjbr-2021-0011

[49] Laban, G., Kappas, A., Morrison, V., Cross, E.S.: OSF Repository: Socially Assistive Robots in Prolonged Interactions for Measuring Expression in Natural Settings (2020). doi $10.17605 / O S F . I O / M 74 C B$ URL https: $/ / \mathrm{osf} . \mathrm{io} / \mathrm{m} 74 \mathrm{cb} /$

[50] Ledbetter, A.M.: Measuring Online Communication Attitude: Instrument Development and Validation. Communication Monographs 76(4), $463-486$ (2009). doi 10.1080/03637750903300262. URL https://doi.org/10. 1080/03637750903300262

[51] Leite, I., Martinho, C., Paiva, A.: Social Robots for Long-Term Interaction: A Survey. International Journal of Social Robotics 5(2), 291-308 (2013). doi 10.1007/s12369-013-0178-y. URL https://doi.org/10.1007/ s12369-013-0178-y

[52] Levinson, L., Gvirsman, O., Gorodesky, I.M., Perez, A., Gonen, E., Gordon, G.: Learning in Summer Camp with Social Robots: A Morphological Study. International Journal of Social Robotics (2020). doi:10.1007/s12369-02000689-y. URL https://doi.org/10.1007/s12369-020-00689-y

[53] Logacjov, A., Kerzel, M., Wermter, S.: Learning Then, Learning Now, and Every Second in Between: Lifelong Learning With a Simulated Humanoid Robot. Frontiers in Neurorobotics 15, 78 (2021). doi:10.3389/FNBOT.2021.669534. URL https://www.frontiersin.org/articles/10.3389/fnbot $2021.669534 /$ full

[54] van Maris, A., Zook, N., Caleb-Solly, P., Studley, M., Winfield, A., Dogramadzi, S.: Designing Ethical Social Robots-A Longitudinal Field Study With Older Adults. Frontiers in Robotics and AI 7, 1 (2020). doi:10.3389/FROBT.2020.00001

[55] Michaelis, J.E., Mutlu, B.: Someone to Read with: Design of and Experiences with an In-Home Learning Companion Robot for Reading. Proceedings of the 2017 CHI Conference on Human Factors in Computing Systems (2017). doi:10.1145/3025453. URL http://dx.doi.org/10.1145/3025453.3025499

[56] Michaelis, J.E., Mutlu, B.: Supporting Interest in Science Learning with a Social Robot. Proceedings of the 18th ACM International Conference on Interaction Design and Children (2019). doi:10.1145/3311927. URL https://doi.org/10.1145/3311927.3323154

[57] Munafò, M.R., Nosek, B.A., Bishop, D.V.M., Button, K.S., Chambers, C.D., du Sert, N.P., Simonsohn, U., Wagenmakers, E.J., Ware, J.J., Ioannidis, J.P.A.: A manifesto for reproducible science. Nature Human Behaviour 1(1), 21 (2017). doi 10.1038/s41562-016-0021

[58] Nahum, M., Van Vleet, T.M., Sohal, V.S., Mirzabekov, J.J., Rao, V.R., Wallace, D.L., Lee, M.B., Dawes, H., Stark-Inbar, A., Jordan, J.T., Biagianti, B., Merzenich, M., Chang, E.F.: Immediate Mood Scaler: Tracking Symptoms of Depression and Anxiety Using a Novel Mobile Mood Scale. JMIR Mhealth Uhealth 5(4), e44 
(2017). doi:10.2196/mhealth.6544. URL http://mhealth.jmir.org/2017/4/e44/https://doi.org/10. 2196/mhealth.6544http://www.ncbi.nlm.nih.gov/pubmed/28404542

[59] Nelson, L.D., Simmons, J.P., Simonsohn, U.: Let's Publish Fewer Papers. Psychological Inquiry 23(3), 291-293 (2012). doi $10.1080 / 1047840 X .2012 .705245$

[60] Nomura, T., Kanda, T., Yamada, S., Suzuki, T.: The effects of assistive walking robots for health care support on older persons: a preliminary field experiment in an elder care facility. Intelligent Service Robotics 2021 14:1 14(1), 25-32 (2021). doi:10.1007/S11370-020-00345-4. URL https://link.springer.com/article/10. 1007/s11370-020-00345-4

[61] Petty, R.E., Mirels, H.L.: Intimacy and Scarcity of Self-Disclosure: Effects on Interpersonal Attraction for Males and Females. Personality and Social Psychology Bulletin 7(3), 493-503 (1981). doi:10.1177/014616728173020 URL https://doi.org/10.1177/014616728173020

[62] Riddoch, K.A., Cross, E.S.: "Hit the Robot on the Head With This Mallet" - Making a Case for Including More Open Questions in HRI Research. Frontiers in Robotics and AI 8, 2 (2021). doi 10.3389/frobt.2021.603510 URL www.frontiersin.org

[63] Robinson, N.L., Connolly, J., Hides, L., Kavanagh, D.J.: A Social Robot to Deliver an 8-Week Intervention for Diabetes Management: Initial Test of Feasibility in a Hospital Clinic. Lecture Notes in Computer Science (including subseries Lecture Notes in Artificial Intelligence and Lecture Notes in Bioinformatics) 12483 LNAI, 628-639 (2020). doi:10.1007/978-3-030-62056-1_52. URL https://link.springer.com/chapter/10. 1007/978-3-030-62056-1_52

[64] Robinson, N.L., Connolly, J., Hides, L., Kavanagh, D.J.: Social robots as treatment agents: Pilot randomized controlled trial to deliver a behavior change intervention. Internet Interventions 21, 100320 (2020). doi https://doi.org/10.1016/j.invent.2020.100320. URL http://www.sciencedirect.com/science/ article/pii/S2214782919301241

[65] Robinson, N.L., Cottier, T.V., Kavanagh, D.J.: Psychosocial Health Interventions by Social Robots: Systematic Review of Randomized Controlled Trials. J Med Internet Res 21(5), 1-20 (2019). doi:10.2196/13203

[66] Saucier, G.: Mini-Markers: A brief version of Goldberg's unipolar Big-Five markers. Journal of personality assessment 63(3), 506-516 (1994). doi:10.1207/s15327752jpa6303_8

[67] Scassellati, B., Vázquez, M.: The potential of socially assistive robots during infectious disease outbreaks. Science Robotics 5(44) (2020). doi:10.1126/scirobotics.abc9014. URL https://robotics.sciencemag.org/ content/5/44/eabc9014https://robotics.sciencemag.org/content/5/44/eabc9014.abstract

[68] Scoglio, A.A.J., Reilly, E.D., Gorman, J.A., Drebing, C.E.: Use of Social Robots in Mental Health and Well-Being Research: Systematic Review. J Med Internet Res 21(7), e13322 (2019). doi 10.2196/13322

[69] Simmons, J.P., Nelson, L.D., Simonsohn, U.: False-Positive Psychology: Undisclosed Flexibility in Data Collection and Analysis Allows Presenting Anything as Significant. Psychol Sci 22(11), 1359-1366 (2011). doi: $10.1177 / 0956797611417632$

[70] Smedegaard, C.V.: Reframing the Role of Novelty within Social HRI: From Noise to Information. In: Proceedings of the 14th ACM/IEEE International Conference on Human-Robot Interaction, HRI '19, p. 411-420. IEEE Press (2019). doi:10.1109/HRI.2019.8673219

[71] Spaulding, S., Shen, J., Park, H.W., Breazeal, C.: Lifelong Personalization via Gaussian Process Modeling for Long-Term HRI. Frontiers in Robotics and AI 8, 152 (2021). doi 10.3389/FROBT.2021.683066. URL www.frontiersin.org

[72] Stower, R., Kappas, A.: "Oh no, my instructions were wrong!" An Exploratory Pilot Towards Children's Trust in Social Robots. In: 2020 29th IEEE International Conference on Robot and Human Interactive Communication (RO-MAN), pp. 641-646 (2020). doi:10.1109/RO-MAN47096.2020.9223495 
[73] Tanaka, F., Cicourel, A., Movellan, J.R.: Socialization between toddlers and robots at an early childhood education center. Proceedings of the National Academy of Sciences of the United States of America 104(46), 17954-17958 (2007). doi:10.1073/PNAS.0707769104

[74] Taylor, S., Landry, C.A., Paluszek, M.M., Fergus, T.A., McKay, D., Asmundson, G.J.G.: Development and initial validation of the COVID Stress Scales. Journal of Anxiety Disorders 72, 102232 (2020). doi:https://doi.org/10.1016/j.janxdis.2020.102232. URL https://www.sciencedirect.com/ science/article/pii/S0887618520300463

[75] Woo, H., LeTendre, G.K., Pham-Shouse, T., Xiong, Y.: The use of social robots in classrooms: A review of field-based studies. Educational Research Review 33, 100388 (2021). doi:10.1016/j.edurev.2021.100388

[76] Yang, G.Z., Nelson, B.J., Murphy, R.R., Choset, H., Christensen, H., Collins, S.H., Dario, P., Goldberg, K., Ikuta, K., Jacobstein, N., Kragic, D., Taylor, R.H., McNutt, M.: Combating COVID-19-The role of robotics in managing public health and infectious diseases. Science Robotics 5(40), 5589 (2020). doi:10.1126/SCIROBOTICS.ABB5589. URL https://robotics.sciencemag.org/content/5/ 40/eabb5589https://robotics.sciencemag.org/content/5/40/eabb5589.abstract 